\title{
ARRAY CONVERGENCE OF FUNCTIONS OF THE FIRST BAIRE CLASS
}

\author{
HELMUT KNAUST
}

(Communicated by William J. Davis)

\begin{abstract}
We show that every array $(x(i, j): 1 \leq i<j<\infty)$ of elements in a pointwise compact subset of the Baire-1 functions on a Polish space, whose iterated pointwise $\operatorname{limit}_{\lim _{i}} \lim _{j} x(i, j)$ exists, is converging Ramsey-uniformly. An array $\left(x(i, j)_{i<j}\right)$ in a Hausdorff space $\mathscr{T}$ is said to converge Ramseyuniformly to some $x$ in $\mathscr{T}$, if every subsequence of the positive integers has a further subsequence $\left(m_{i}\right)$ such that every open neighborhood $U$ of $x$ in $\mathscr{T}$ contains all elements $x\left(m_{i}, m_{j}\right)$ with $i<j$ except for finitely many $i$.
\end{abstract}

\section{INTRODUCTION}

It is a well-known consequence of Ramsey's theorem that every array $\left(a_{i j}\right)_{i<j}$ of real numbers with $\lim _{i} \lim _{j} a_{i j}=a$ for some $a \in \mathbb{R}$ has the following property: There is a subsequence $\left(m_{i}\right)$ so that for all $\varepsilon>0$ there is an $n \in \mathbb{N}$ such that $\left|a_{m_{i} m_{j}}-a\right|<\varepsilon$ for all $n<m_{i}<m_{j}$. This result generalizes easily to Hausdorff spaces which satisfy the first countability axiom.

The purpose of our note is to show that a corresponding result holds for the space of functions of the first Baire class $\mathscr{B}_{1}(\Omega)$ on a Polish space $\Omega$, given the topology of pointwise convergence.

Let us say that an array $(x(i, j): 1 \leq i<j<\infty)$ of elements in a Hausdorff space $\mathscr{T}$ converges Ramsey-uniformly to some $x \in \mathscr{T}$, if every subsequence of $\mathbb{N}$ has a further subsequence $\left(m_{i}\right)$ such that for every open neighborhood $U$ of $x$ in $\mathscr{T}$ there is an $n \in \mathbb{N}$ so that $x\left(m_{i}, m_{j}\right) \in U$ for all $n<m_{i}<m_{j}$.

With this notation we can state our main result as follows:

Theorem 1. Let $\Omega$ be a Polish space and let $K$ be a pointwise compact subset of $\mathscr{B}_{1}(\Omega)$. If $x$ and $(x(i, j))_{i<j}$ are elements in $K$ with $\lim _{i} \lim _{j} x(i, j)=x$, then $(x(i, j))$ converges Ramsey-uniformly to $x$.

A topological space $\Omega$ is Polish, if it is homeomorphic to a complete separable metric space. A real-valued function is of the first Baire class on $\Omega$, if it is the pointwise limit of a sequence of continuous functions on $\Omega$.

Received by the editors December 20, 1989 and, in revised form, May 31, 1990.

1980 Mathematics Subject Classification (1985 Revision). Primary 46E15; Secondary 46B20.

Key words and phrases. First Baire class, array convergence, Ramsey theory. 
It is a fundamental result of Bourgain, Fremlin, and Talagrand [2] that $\mathscr{B}_{1}(\Omega)$ is an angelic space, if $\Omega$ is Polish. A Hausdorff space $\mathscr{T}$ is angelic, if for every relatively compact subset $A$ of $\mathscr{T}$ each point in the closure of $A$ is the limit of a sequence in $A$ and if relatively countably compact sets in $\mathscr{T}$ are relatively compact. In angelic spaces the notions of (relative) compactness, (relative) countable compactness, and (relative) sequential compactness coincide. Further basic results about angelic spaces can be found in [7].

Theorem 1 strengthens-in the case of functions of the first Baire class on a Polish space-a result of Boehme and Rosenfeld [1, Theorem 1], which we phrase for our purposes as follows:

Lemma 2. Let $\mathscr{T}$ be an angelic space, and let $x$ and $(x(i, j))_{i<j}$ be elements in a compact subset of $\mathscr{T}$ with $\lim _{i} \lim _{j} x(i, j)=x$. Then there is a subsequence $\left(m_{i}\right)$ of $\mathbb{N}$ with $\lim _{k} x\left(m_{2 k-1}, m_{2 k}\right)=x$.

Lemma 2 was also obtained independently, in the $\mathscr{B}_{1}(\Omega)$-setting, by Rosenthal [8, Theorem 3.6].

From Theorem 1 and a result by Odell and Rosenthal [6] we obtain the following Banach space corollary:

Corollary 3. Let $X$ be a separable Banach space not containing $l_{1}$. If $x^{* *}$ and $\left(x^{* *}(i, j)\right)_{i<j}$ are elements in a bounded subset of $X^{* *}$ with

$$
\omega^{*}-\lim _{i} \omega^{*}-\lim _{j} x^{* *}(i, j)=x^{* *},
$$

then $\left(x^{* *}(i, j)\right)$ converges Ramsey-uniformly to $x^{* *}$ in the $\omega^{*}$-topology.

The proof of Theorem 1 utilizes Lemma 2 to extract "nice" converging subsequences out of the given array $(x(i, j))$. We use Ramsey theory to produce the subarray for which one obtains Ramsey-uniform convergence.

If $M$ is an infinite subset of $\mathbb{N}, \mathscr{P}^{\infty}(M)$ will denote the set of all infinite subsets of $M$. We give $\mathscr{P}^{\infty}(\mathbb{N})$ the topology, which is inherited by considering $\mathscr{P}^{\infty}(\mathbb{N})$ as a subspace of $\{0,1\}^{\mathbb{N}}$ endowed with the product topology.

A subset $\mathscr{A} \subset \mathscr{P}^{\infty}(\mathbb{N})$ is called a Ramsey set, if for all $L \in \mathscr{P}^{\infty}(\mathbb{N})$ there is an $M \in \mathscr{P}^{\infty}(L)$ such that either $\mathscr{P}^{\infty}(M) \subset \mathscr{A}$ or $\mathscr{P}^{\infty}(M) \cap \mathscr{A}=\varnothing$. It is known that analytic (and coanalytic) subsets of $\mathscr{P}^{\infty}(\mathbb{N})$ are Ramsey sets [3, 9]. For a proof of this result, some history and more general results, see [5].

I would like to thank D. Alspach, E. Odell, and H. P. Rosenthal for useful discussions.

\section{Proof}

Proof of Theorem 1. Let $x$ and $x(i, j)$ with $1 \leq i<j<\infty$ be elements in $K$ such that $\lim _{i} \lim _{j} x(i, j)=x$. We let

$$
\mathscr{A}=\left\{M=\left(m_{i}\right) \in \mathscr{P}^{\infty}(\mathbb{N}):\left(x\left(m_{2 k-1}, m_{2 k}\right)\right)_{k=1}^{\infty} \text { is pointwise convergent }\right\} .
$$

Lemma 4. $\mathscr{A}$ is coanalytic. 
We postpone the proof of the lemma and proceed with the proof of the theorem. Since $\mathscr{A}$ is coanalytic, $\mathscr{A}$ is a Ramsey set. Let $L \in \mathscr{P}^{\infty}(\mathbb{N})$. We can thus find $M=\left(m_{i}\right)_{i=1}^{\infty} \in \mathscr{P}^{\infty}(L)$ so that $\mathscr{P}^{\infty}(M) \subset \mathscr{A}$ or $\mathscr{P}^{\infty}(M) \cap \mathscr{A}=\varnothing$. Lemma 2 shows that the first alternative holds. Moreover, Lemma 2 asserts that $\lim _{k} x\left(m_{2 k-1}^{\prime}, m_{2 k}^{\prime}\right)=x$ for some $M^{\prime}=\left(m_{i}^{\prime}\right) \in \mathscr{P}^{\infty}(M)$.

Suppose now the conclusion of Theorem 1 fails for $M^{\prime}$. Then there is an open neighborhood $U$ of $x$ and a subsequence $M^{\prime \prime} \subset M^{\prime}$ with

$$
x\left(m_{2 k-1}^{\prime \prime}, m_{2 k}^{\prime \prime}\right) \notin U \text { for all } k \in \mathbb{N} .
$$

Since $M^{\prime \prime} \in \mathscr{P}^{\infty}(M)$, we have $M^{\prime \prime} \in \mathscr{A}$ and thus $\lim _{k} x\left(m_{2 k-1}^{\prime \prime}, m_{2 k}^{\prime \prime}\right)=y$ for some $y \in \mathscr{B}_{1}(\Omega)$. Note that $y \neq x$.

We now construct a subsequence $N=\left(n_{i}\right) \in \mathscr{P}^{\infty}(M)$ inductively as follows: Let $n_{1}=m_{1}^{\prime}$ and $n_{2}=m_{2}^{\prime}$. Once $n_{1}, n_{2}, \ldots, n_{2 k}$ have been chosen, we define $n_{2 k+1}$ and $n_{2 k+2}$ as follows: If $k$ is odd, we choose an $l \in \mathbb{N}$ so that $m_{2 l-1}^{\prime \prime}>n_{2 k}$ and let $n_{2 k+1}=m_{2 l-1}^{\prime \prime}, n_{2 k+2}=m_{2 l}^{\prime \prime}$. If $k$ is even, we can find an $l \in \mathbb{N}$ with $m_{2 l-1}^{\prime}>n_{2 k}$ and then let $n_{2 k+1}=m_{2 l-1}^{\prime}, n_{2 k+2}=m_{2 l}^{\prime}$. On the one hand the sequence $\left(x\left(n_{2 k-1}, n_{2 k}\right)\right)$ is pointwise convergent, on the other hand it contains two subsequences converging to $x$ and $y$ respectively. This yields a contradiction.

Proof of Lemma 4. The proof of Lemma 4 uses techniques similar to those employed in [10].

Let $Y$ be the set of all real-valued arrays $(a(i, j))_{i<j}$, endowed with the topology of pointwise convergence. We set $Z=\mathscr{P}^{\infty}(\mathbb{N}) \times Y$ and denote by $\phi: \Omega \rightarrow Y$ the canonical map defined by $\phi(\omega)=(x(i, j)(\omega))_{i<j}$. Since $\phi$ is a Borel-measurable map and $\Omega$ is Polish, $\phi(\Omega)$ is analytic in $Y$ (see $[4, \S 38]$ ). Consequently $Z_{1}:=\mathscr{P}^{\infty}(\mathbb{N}) \times \phi(\Omega)$ is analytic in $Z$.

We define a set $Z_{2} \subset \mathscr{P}^{\infty}(\mathbb{N}) \times Y$ as follows:

$$
Z_{2}=\left\{(M,(a(i, j))):\left(a\left(m_{2 k-1}, m_{2 k}\right)\right)_{k=1}^{\infty} \text { is not Cauchy }\right\} .
$$

Observing that the set

$$
\begin{aligned}
Z_{2}^{l, N}:=\{( & (M,(a(i, j))): \text { there are } k_{1}, k_{2}>N \text { with } \\
& \left.\left|a\left(m_{2 k_{1}-1}, m_{2 k_{1}}\right)-a\left(m_{2 k_{2}-1}, m_{2 k_{2}}\right)\right|>2^{-l}\right\}
\end{aligned}
$$

is open, and that

$$
Z_{2}=\bigcup_{l \in \mathbb{N}} \bigcap_{N \in \mathbb{N}} Z_{2}^{l, N}
$$

we obtain that $Z_{2}$ is a $G_{\delta \sigma}$-set in $Z$.

Consequently $Z_{1} \cap Z_{2}$ is analytic in $Z$. We let $P: Z \rightarrow \mathscr{P}^{\infty}(\mathbb{N})$ be the projection of $Z$ onto its first coordinate. One can see easily that the complement of $\mathscr{A}$ is equal to $P\left(Z_{1} \cap Z_{2}\right)$. Thus $\mathscr{P}^{\infty}(\mathbb{N}) \backslash \mathscr{A}$ is analytic in $\mathscr{P}^{\infty}(\mathbb{N})$ as the continuous image of an analytic set in $Z$ (see $[4, \S 38]$ ). 
Problem. Does Theorem 1 hold for arbitrary angelic spaces? Lemma 2 reduces this problem to the apparently open question, whether the set $\mathscr{A} \subset \mathscr{P}^{\infty}(\mathbb{N})$, defined at the beginning of the proof, is still a Ramsey set for arbitrary angelic spaces.

\section{REFERENCES}

1. T. K. Boehme and M. Rosenfeld, An example of two compact Hausdorff Fréchet spaces whose product is not Fréchet, J. London Math. Soc. 8 (1974), 339-344.

2. J. Bourgain, D. Fremlin, and M. Talagrand, Pointwise compact sets of Baire-measurable functions, Amer. J. Math. 100 (1978), 845-886.

3. E. E. Ellentuck, A new proof that analytic sets are Ramsey, J. Symbolic Logic 39 (1974), 163-165.

4. K. Kuratowski, Topology, Academic Press, New York, 1966.

5. E. Odell, Applications of Ramsey theorems to Banach space theory, Notes in Banach Spaces (H. E. Lacey, ed.), Univ. Texas Press, Austin, 1980, pp. 379-404.

6. E. Odell and H. P. Rosenthal, $A$ double dual characterization of separable Banach spaces containing $l^{1}$, Israel J. Math. 20 (1975), 375-384.

7. J. D. Pryce, A device of R. J. Whitley's applied to pointwise compactness in spaces of continuous functions, Proc. London Math. Soc. 23 (1971), 532-546.

8. H. P. Rosenthal, Some remarks concerning unconditional basic sequences, Longhorn Notes 1982-83, The University of Texas, Austin, pp. 15-48.

9. J. Silver, Every analytic set is Ramsey, J. Symbolic Logic 35 (1970), 60-64.

10. J. Stern, A Ramsey theorem for trees, with an application to Banach spaces, Israel J. Math. 29 (1978), 179-188.

Department of Mathematics, Oklahoma State University, Stillwater, Oklahoma 